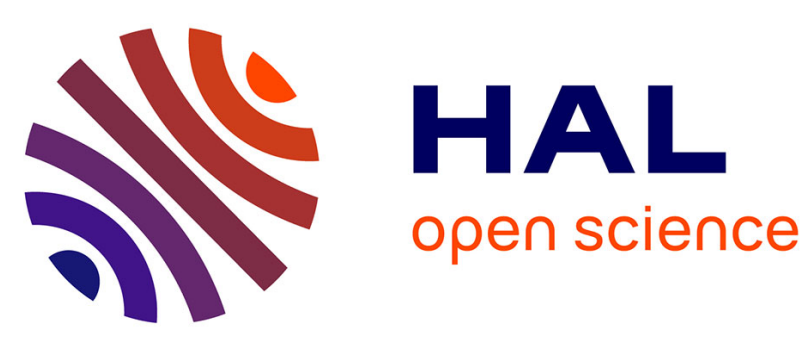

\title{
Memory Efficient Self-Stabilizing k-Independent Dominating Set Construction
}

Colette Johnen

\section{To cite this version:}

Colette Johnen. Memory Efficient Self-Stabilizing k-Independent Dominating Set Construction. Third International Conference on Networked Systems - NETYS 2015, May 2015, Agadir, Morocco. 10.1007/978-3-319-26850-7_24. hal-00843995v2

\section{HAL Id: hal-00843995 \\ https://hal.science/hal-00843995v2}

Submitted on 1 Oct 2013

HAL is a multi-disciplinary open access archive for the deposit and dissemination of scientific research documents, whether they are published or not. The documents may come from teaching and research institutions in France or abroad, or from public or private research centers.
L'archive ouverte pluridisciplinaire HAL, est destinée au dépôt et à la diffusion de documents scientifiques de niveau recherche, publiés ou non, émanant des établissements d'enseignement et de recherche français ou étrangers, des laboratoires publics ou privés. 


\title{
Memory efficient Self-Stabilizing $k$-Independent Dominating Set Construction* Labri Technical Report RR-1473-13
}

\author{
Colette Johnen \\ Univ. Bordeaux, LaBRI, UMR 5800, F-33400 Talence, France
}

October 1, 2013

\begin{abstract}
We propose a memory efficient self-stabilizing protocol building $k$-independent dominating sets. A $k$-independent dominating set is a $k$-independent set and a $k$ dominating set. A set of nodes, $I$, is $k$-independent if the distance between any pair of nodes in $I$ is at least $k+1$. A set of nodes, $D$, is a $k$-dominating if every node is within distance $k$ of a node of $D$.

Our algorithm, named $\mathcal{S I D}$, is silent; it converges under the unfair distributed scheduler (the weakest scheduling assumption).

We established that any $k$-independent sets contains at most $\lfloor(2 n) /(k+2)\rfloor$ nodes, $n$ being the network size.

The protocol $\mathcal{S I D}$ is memory efficient : it requires only $2 \log ((k+1) n+1)+1$ bits per node.

The correctness and the termination of the protocol $\mathcal{S I D}$ is proven.

The computation of the convergence time of the protocol $\mathcal{S I D}$ is opened question.

keywords distributed computing, fault tolerance, self-stabilization, $k$-dominating set, $k$-independent set, $k$-independent dominating set, memory efficient
\end{abstract}

\section{Introduction}

The clustering of networks consists of partitioning network nodes into non-overlapping groups called clusters. Each cluster has a single head, called leader, that acts as local coordinator of the cluster, and eventually a set of standard nodes. In 1-hop clusters, the standard nodes are neighbor (at distance 1) of their leader. Clustering is found very attractive in infrastructure-less networks, like ad-hoc networks, since it limits the responsibility of network management only to leaders, and it allows the use of hierarchical routing. This is why numerous self-stabilizing 1-hop clustering protocols were proposed in the literature $[9,10,8,11,13,14,15,16,19,20]$.

${ }^{*}$ This work was partially supported by the ANR project Displexity. 
Silent self-stabilizing protocols building $k$-hops clustering set are proposed for $k>1$. In $k$-hop clusters, the distance between a standard node and its leader is at most $k$ [1, 2, 3, 7]. The sets of cluster heads built by these protocols are not $k$-independent. The protocol of [1] is designed for $k=2$. Routing tables are maintained by the cluster heads to store routing information to nodes both within and outside the cluster. The goal of the protocol in [2] is to build bounded size clusters (each cluster has at most Cluster_Max nodes). bits per node. The protocol of [3] is designed for weighted edges networks; it requires at least $O(k \cdot \log (n))$ bits per node. The protocol of [7] requires at least $2(k+1) \log (n)$

In [17, 18, Larsson and Tsigas propose self-stabilizing $(l, k)$-clustering protocols under various assupmtions. These protocols ensure, if possible, that each node has $l$ clusterheads at distance at most $k$.

Related Works. In [4], a silent self-stabilizing protocol extracting a minimal $k$-dominating set from any $k$-dominating set is proposed. A minimal $k$-dominating set has no proper subset which also a $k$-dominating set. The protocol requires at least $O(k \cdot \log (n))$ bits per node.

The paper [6] presents a silent self-stabilizing protocol building a small $k$-dominating set : the obtained dominating set contains at most $\lceil n /(k+1)\rceil$ nodes. The protocol of [6] requires $O(\log (n)+k \cdot \log (n / k))$ bits per node. The protocol of [5] builds competitive $k$-dominating sets : the obtained dominating set contains at most $1+\lfloor(n-1) /(k+1)\rfloor$ nodes. The protocol of [5] requires $O(\log (2 k \cdot 2(\Delta+1) .2 n . D))$ bits per node, where $D$ is the network diameter, and $\Delta$ is a bound on node degree. These both protocols use the hierachical collateral composition of several silent self-stabilizing protocols whose a leader election protocol and a spanning tree construction rooted to the elected leader. So they require more memory space than our protocol.

In [12, a fast silent self-stabilizing protocol building a $k$-independent dominating set is proposed the protocol converges in $4 n+k$ rounds and it requires $(k+1) \log (n+1)$ bits per node. The computation of the convergence time of the protocol $\mathcal{S I D}$ is opened question.

Contribution. In this paper, we consider the problem of computing a $k$-independent dominating set in a self-stabilizing manner in case where $k>1$. A nodes set is $k$ independent dominating set (also called maximal $k$-independent set) if and only if this set is a $k$-independent set and a $k$-dominating set. A set of nodes, $I$ is $k$-independent if the distance between any pair of $I$ 's nodes is at least $k+1$. A set of nodes $D$ is $k$-dominating if every node is within distance $k$ of a node of $D$.

The presented protocol, named $\mathcal{S I D}$, is simple : no use of the the hierachical collateral composition, no need of leader election process, neither the building of spanning tree. The protocol $\mathcal{S I D}$ converges under the unfair distributed scheduler (the weakest scheduling assumption). The algorithm $\mathcal{S I D}$ is silent. The protocol $\mathcal{S I D}$ is memory efficient : it requires only $2 \log ((k+1) n+1)+1$ bits per node. 
In section 2, we establish that any $k$-independent sets contain at most $\lfloor(2 n) /(k+2)\rfloor$ nodes, $n$ being the network size. So the protocol of [12] and the protocol $\mathcal{S I D}$ have the same upper bound on the size of built $k$ independent dominating sets : $\lfloor(2 n) /(k+2)\rfloor$ nodes.

Paper outline. The rest of the paper is organised as follows. In section 2, communication and computation models are defined. The protocol $\mathcal{S I D}$ is presented in section 3. In the section 4, the correctness of the $\mathcal{S I D}$ protocol is proven. The termination of the $\mathcal{S I D}$ protocol is established in the section 5 .

\section{Model and Concepts}

A distributed system $S$ is an undirected graph $G=(V, E)$ where vertex set $V$ is the set of nodes and edge set $E$ is the set of communication links. A link $(u, v) \in E$ if and only if $u$ and $v$ can directly communicate (links are bidirectional); so, $u$ and $v$ are neighbors. We denote by $N_{v}$ the set of $v$ 's neighbors: $N_{v}=\{u \in V \mid(u, v) \in E\}$. The distance between the nodes $u$ and $v$ is denoted $\operatorname{dist}(u, v)$. k-neigborhood $(v)=$ $\{u \in V \mid \operatorname{dist}(u, v) \in[1, k]\}$.

\section{Definition 1 ( $k$-independent dominating set)}

Let $D$ be a subset of $V ; D$ is a $k$-dominating set if and only if $\forall v \in V / D$ we have $\mathrm{k}$-neigborhood $(v) \cap D \neq \emptyset$.

Let $I$ be a subset of $V ; I$ is a $k$-independent set if and only if $\forall u \in I$ we have $\mathrm{k}$-neigborhood $(u) \cap I=\emptyset$.

$A$ subset of $V$ is a distance- $k$ independent dominating set if this subset is a distance- $k$ dominating set and a distance- $k$ independent set.

Lemma 1 The size of a $k$-independent set is at most $\max (\lfloor(2 n) /(k+2)\rfloor, 1)$.

Proof 1 Let I be a k-independent set such that $|I|>1$. Let $v$ be a node of $I$. We denote by closer $(v)$ the set of nodes closer to $v$ than any other node of $I$.

Notice that $\bigcup_{w \in I} \operatorname{closer}(w) \subset V$ and $\operatorname{closer}(v) \cap \operatorname{closer}(u)=\emptyset, \forall(u, v) \in I^{2}$. Let $u$ be the closest node to $v$ that belongs to $I$. Let $x$ be node on the path from $v$ to $u$ such that $0 \leq \operatorname{dist}(v, x) \leq\lfloor k / 2\rfloor$. Let $w$ be a node of $I$ other than $v$. We have $\operatorname{dist}(w, x)>k-\operatorname{dist}(v, x) \geq\lfloor k / 2\rfloor$ because $k<\operatorname{dist}(w, v) \leq \operatorname{dist}(v, x)+\operatorname{dist}(x, w)$. So, closer $(v)$ contains the first $\lfloor k / 2\rfloor+1$ nodes in the path from $v$ to $u$. We conclude that $|I| \leq\lfloor(2 n) /(k+2)\rfloor$.

The protocol $\mathcal{S I D}$ builds $k$-independent sets. So, the obtained $k$-independent dominating set contains at most $\lfloor(2 n) /(k+2)\rfloor$ nodes.

Furthermore, at every node $v$ in the network is assigned an identifier, denoted by $i d_{v}$. Two distinct nodes have different identifier. It is possible to order the identifier values. The symbol $\perp$ denotes a value smaller than any identifier value in the network. 
Each node $v$ maintains a set of shared variables such that $v$ can read its own variables and those of its neighbors, but it can modify only its variables. The state of a node is defined by the values of its local variables. The cartesian product of states of all nodes determines the configuration of the system. The program of each node is a set of rules. Each rule has the form: Rule $_{i}:<$ Guard $_{i}>\longrightarrow<$ Action $_{i}>$. The guard of a v's rule is a boolean expression involving the state of the node $v$, and those of its neighbors. The action of a $v$ 's rule updates $v$ 's state. A rule can be executed only if it is enabled, i.e., its guard evaluates to true. A node is said to be enabled if at least one of its rules is enabled. In a terminal configuration, no node is enabled.

During a computation step $c_{i} \rightarrow c_{i+1}$, one or several enabled nodes perform an enabled action and the system reaches the configuration $c_{i+1}$ from $c_{i}$. A computation $e$ is a sequence of configurations $e=c_{0}, c_{1}, \ldots, c_{i}, \ldots$, where $c_{i+1}$ is reached from $c_{i}$ by one computation step: $\forall i \geqslant 0, c_{i} \rightarrow c_{i+1}$. We say that a computation $e$ is maximal if it is infinite, or if it reaches a terminal configuration. We note by $\mathcal{C}$ the set of all possible configurations, and by $\mathcal{E}$ the set of all maximal computations. The set of maximal computations starting from a particular configuration $c \in \mathcal{C}$ is denoted $\mathcal{E}_{c}$. $\mathcal{E}_{A}$ denotes the set of all maximal computations where the initial configuration belongs to the set of configurations $A \subset \mathcal{C}$.

Definition 2 (Attractor) Let $B_{1}$ and $B_{2}$ be subsets of $\mathcal{C}$. $B_{2}$ is an attractor from $B_{1}$, if and only if the following conditions hold:

- Convergence: $\forall c \in B_{1}$, If $\left(\mathcal{E}_{c}=\emptyset\right)$ then $c \in B_{2}$ otherwise $\forall e \in \mathcal{E}_{B_{1}}\left(e=c_{1}, c_{2}, \ldots\right), \exists i \geqslant 1, c_{i} \in B_{2}$;

- Closure: $\forall e \in \mathcal{E}_{B_{2}}\left(e=c_{1}, \ldots\right), \forall i \geqslant 1: c_{i} \in B_{2}$.

Definition 3 (Self-stabilization) A distributed system $S$ is self-stabilizing for the specification $\mathcal{S P}$ (a predicate on configurations) if and only if there exists a non-empty set $\mathcal{L} \subseteq \mathcal{C}$, called set of legitimate configurations, such that the following conditions hold:

- $\mathcal{L}$ is an attractor from $\mathcal{C}$;

- Configurations of $\mathcal{L}$ satisfied $\mathcal{S P}$.

A self-stabilizing protocol is silent if all maximal computations are finite.

Stabilization time. We use the round notion to measure the time complexity. The first round of a computation $e=c_{1}, \ldots, c_{j}, \ldots$ is the minimal prefix $e_{1}=c_{1}, \ldots, c_{j}$, such that every enabled node in $c_{1}$ either executes a rule or it is neutralized during a computation step of $e_{1}$. A node $v$ is neutralized during a computation step $c s c_{i} \rightarrow c_{i+1}$, if $v$ is enabled in $c_{i}$ and disabled in $c_{i+1}$, but it did not execute any action during $c s$.

Let $e_{2}$ be the suffix of $e$ such that $e=e_{1} e_{2}$. The second round of $e$ is the first round of $e_{2}$, and so on.

The stabilization time is the number of rounds of a computation reaching a legitimate configuration from any initial one. 


\section{The protocol $\mathcal{S I D}$}

In the following subsection, we gives the notation used by the protocol $\mathcal{S I D}$.

\section{$3.1 \quad k$-augmentedID type}

Definition $4 k$-augmentedID type An k-augmentedID value, $a$, is $\perp$ or an $n$-tuple $(d, x)$ such that $d$ is integer with $0 \leq d \leq k$, and $x$ is a node identifier. Let $a=(d, x)$ be $k$-augmentedID value. We use the following notation a.dist $=d$ and a.id $=x$.

Let $v$ be a node of $V, i d_{v}^{+}$is the following $k$-augmentedID value: $\left(0, i d_{v}\right)$.

Definition 5 The total order relation dom on $k$-augmentedID

- $\operatorname{dom}(a, b)=a$ if $b=\perp$, a.id $<$ b.id or a.id $=$ b.id $\wedge$ a.dist $<$ b.dist, otherwise $\operatorname{dom}(a, b)=b$.

- The $k$-augmented value a1 dominates the $k$-augmented value a 2 if and only if $\operatorname{dom}(a 1, a 2)=a 1$.

- Let $X$ be a finite set of $k$-augmentedID values. $\operatorname{dom}(X)$ is the $k$-augmentedID value belonging to $X$ such that any value of $X$ is dominated by $\operatorname{dom}(X)$ (i.e. $\forall y \in X$ we have $\operatorname{dom}(\operatorname{dom}(X), y)=\operatorname{dom}(X))$.

Definition 6 The total order relation min on $k$-augmentedID

- $\min (a, b)=a$ if $b=\perp$, a.dist $<$ b.dist or a.dist $=$ b.dist $\wedge$ a.id $<$ b.id otherwise $\min (a, b)=b$.

- The $k$-augmented value a 1 is larger than the $k$-augmented value a 2 if and only if $\min (a 1, a 2)=a 2$.

- Let $X$ be a finite set of $k$-augmentedID values. $\min (X)$ is the $k$-augmentedID value belonging to $X$ such that any value of $X$ is larger than $\min (X)$ (i.e. $\forall y \in X$ we have $\min (\min (X), y)=\min (X))$.

The node $u 1$ is closer to the node $v$ than the node $u 2$ iff $\operatorname{dist}(u 1, v))<\operatorname{dist}(u 2, v)$ or $i d_{u 1}<i d_{u 2}$. We have $\min \left(\left(\operatorname{dist}(u 1, v), i d_{u 1}\right),\left(\operatorname{dist}(u 2, v), i d_{u}\right)\right)=\left(\operatorname{dist}(u 1, v) ; i d_{u 1}\right)$.

Definition 7 The operation +1 on $k$-augmentedID is defined as follow : $a+1=a$ if $a=\perp$ or if $a . d i s t=k$ otherwise $a+1=($ a.dist +1, a.id $)$ 
Protocol 1: Variables, Predicates and Rules of the Protocol SID on the node $v$

\section{Shared variables}

- $\mathrm{firstHead}(v)$ and secondHead $(v)$. They take value in $k$-augmentedID

\section{Internal variable}

- beReal a boolean variables used by some macros.

\section{Notation}

- firstAugmentedIdSet $(v)=\{a+1 \in k$-augmentedID $a=\operatorname{firstHead}(u) \vee a=\operatorname{secondHead}(u)$ with $u \in N_{v} \wedge$ a.dist $\left.<k \wedge a . i d \neq i d_{v}\right\}$

- $\operatorname{secondAugmentedIdSet}(v)=\{a \in$ firstAugmentedIdSet $\mid a . i d \neq$ firstHead $(v) . i d\}$

\section{Macros}

- isDefended $(v)$ returns true iff firstAugmentedIdSet $(v) \neq \emptyset$.

- isDominated $(v)$ returns true iff $i d_{v}^{+} \neq \operatorname{dom}\left(\right.$ firstAugmentedIdSet $(v) \cup i d_{v}^{+}$).

- correctFirstHead $(v)$ returns true iff

$$
\operatorname{firstHead}(v)==\min (\operatorname{firstAugmentedIdSet}(v)) \text {. }
$$

- computingFirstHead $(v)$ returns the value of $\min ($ firstAugmentedIdSet $(v)$ ).

- correctSecondHead $(v)$ returns true iff

$$
\operatorname{secondHead}(v)==\min (\operatorname{secondAugmentedIdSet}(v) \cup \perp) \text {. }
$$

- computingsSecondHead $(v)$ returns the value of $\min (\operatorname{secondAugmentedIdSet}(v) \cup \perp$ ).

\section{Predicates}

- $\operatorname{Head}(v) \equiv \mathrm{firstHead}(v)==\left(0, i d_{v}\right)$

- $\operatorname{toResign}(v) \equiv$ isDominated $(v)$

- $\operatorname{toElect}(v) \equiv \neg$ isDefended $(v)$

- headToUpdate $(v) \equiv \operatorname{firstHead}(v) \neq\left(0, i d_{v}\right) \vee \operatorname{secondHead}(v) \neq \perp$

- ordinaryToUpdate $(v) \equiv \neg \operatorname{correctFirstHead}(v) \vee \neg \operatorname{correctSecondHead}(v)$

\section{Rules}

$$
\begin{aligned}
\mathbf{R E}(v): \neg \operatorname{Head}(v) \wedge & \operatorname{toElect}(v) \longrightarrow \operatorname{firstHead}(v):=\left(0, i d_{v}\right) ; \operatorname{secondHead}(v):=\perp ; \\
\mathbf{R U}(v): \neg \operatorname{Head}(v) \wedge & \neg \operatorname{toElect}(v) \wedge \operatorname{ordinaryToUpdate}(v) \longrightarrow \\
& \operatorname{computingFirstHead}(v) ; \operatorname{computingSecondHead}(v) ; \\
\mathbf{R R}(v): \operatorname{Head}(v) \wedge & \operatorname{toResign}(v) \longrightarrow \\
& \operatorname{computingFirstHead}(v) ; \operatorname{computingSecondHead}(v) ; \\
\mathbf{R C}(v): \operatorname{Head}(v) \wedge & \rightarrow \operatorname{toResign}(v) \wedge \operatorname{headToUpdate}(v) \longrightarrow \\
& \quad \operatorname{irstHead}(v):=\left(0, i d_{v}\right) ; \operatorname{secondHead}(v):=\perp ;
\end{aligned}
$$




\subsection{Code of the protocol $\mathcal{S I D}$}

A node $v$ is said to be a head if $\operatorname{firstHead}(v)=i d_{v}^{+}$; otherwise it is an ordinary node. The heads set built by the protocol $\mathcal{S I D}$ (defined in protocol 1 ) is a $k$-independent dominating set. The codes of the macros are defined in the protocols 2 to 7 .

In the figure 1 is presented a terminal configuration having three heads. On the same network is presented another terminal configuration having a single head, in the figure 2 .

The variable $\operatorname{firstHead}(v)$ contains the identifier of the closest head to $v$ (with its distance to $v$ ).

The variable secondHead $(v)$ contains the identifier of the second closest head to $v$ (with its distance to $v$ ) inside its $\mathrm{k}$-neighborhood. If a node $v$ does not have two heads in its $k$-neighborhood then secondHead $(v)$ is set to $\perp$. The execution of the rules $\mathbf{R U}$ or the rules $\mathbf{R C}$ updates the two variables firstHead $(v)$, and $\operatorname{secondHead}(v)$ without changing the status of $v$ (i.e. $v$ stays ordinary or head).

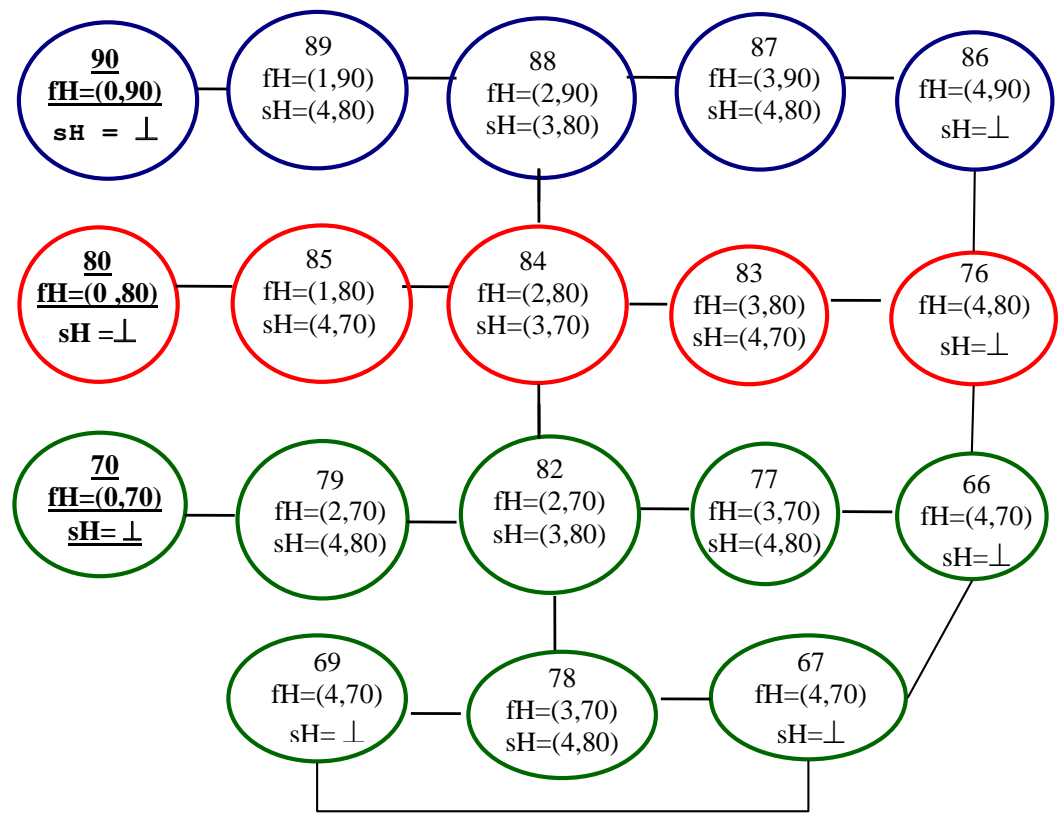

$\mathrm{k}=4$. The head identifiers are underlined. In each node, it is indicated the value of firstHead and the value of secondHead. The color of a node is the color of its closest head.

Figure 1: A terminal configuration of $\mathcal{S I D}$

The macro isDefended $(v)$ (defined in protocol 2 ) returns true if 


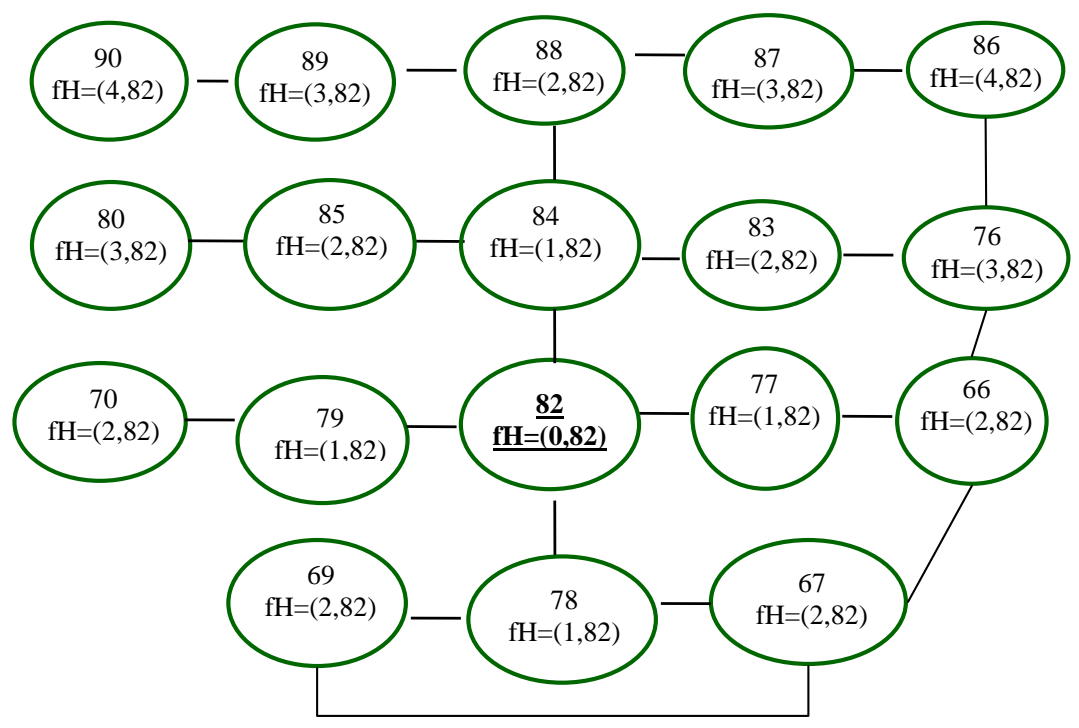

$\mathrm{k}=4$. The head identifier is underlined. In each node, it is indicated the value of firstHead

Figure 2: Another terminal configuration of $\mathcal{S I D}$

the set firstAugmentedIdSet $(v)$ is not empty otherwise the macro returns false.

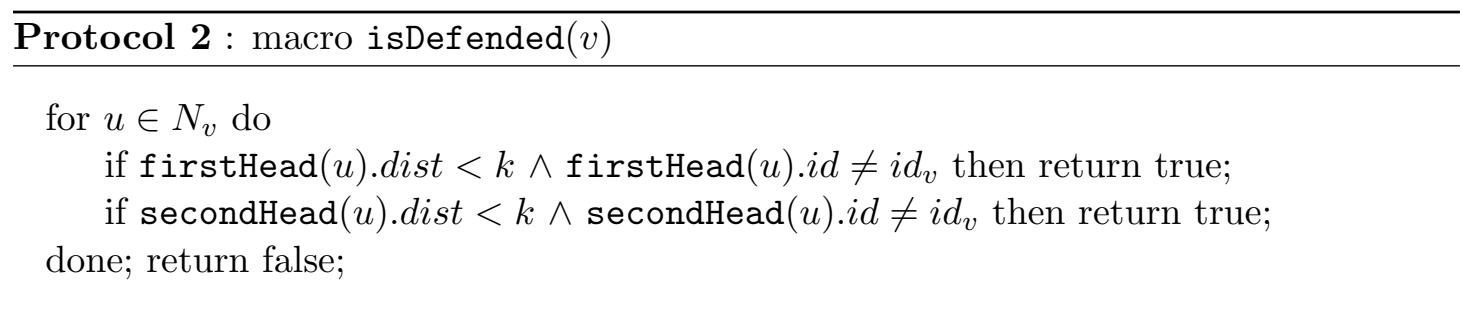

The macro isDominated $(v)$ (defined in protocol 3 ) returns true if a value $x$ of firstAugmentedIdSet $(v)$ dominates the value $i d_{v}^{+}$; otherwise the macro returns false.

The macro correctFirstHead $(v)$ (defined in protocol 4) returns true if the value of $\operatorname{firstHead}(v)$ is $\min (\operatorname{firstAugmentedIdSet}(v)$ ); otherwise the macro returns false. Notice that if the set firstAugmentedIdSet $(v))$ is empty the macro returns false;

The macro computingFirstHead $(v)$ (defined in protocol 5 ) returns $\min ($ firstAugmentedIdSet $(v))$ if the set firstAugmentedIdSet $(v)$ is not empty; otherwise the macro returns $\perp$.

The macro correctsecondHead $(v)$ (defined in protocol 6) returns true if the value of 


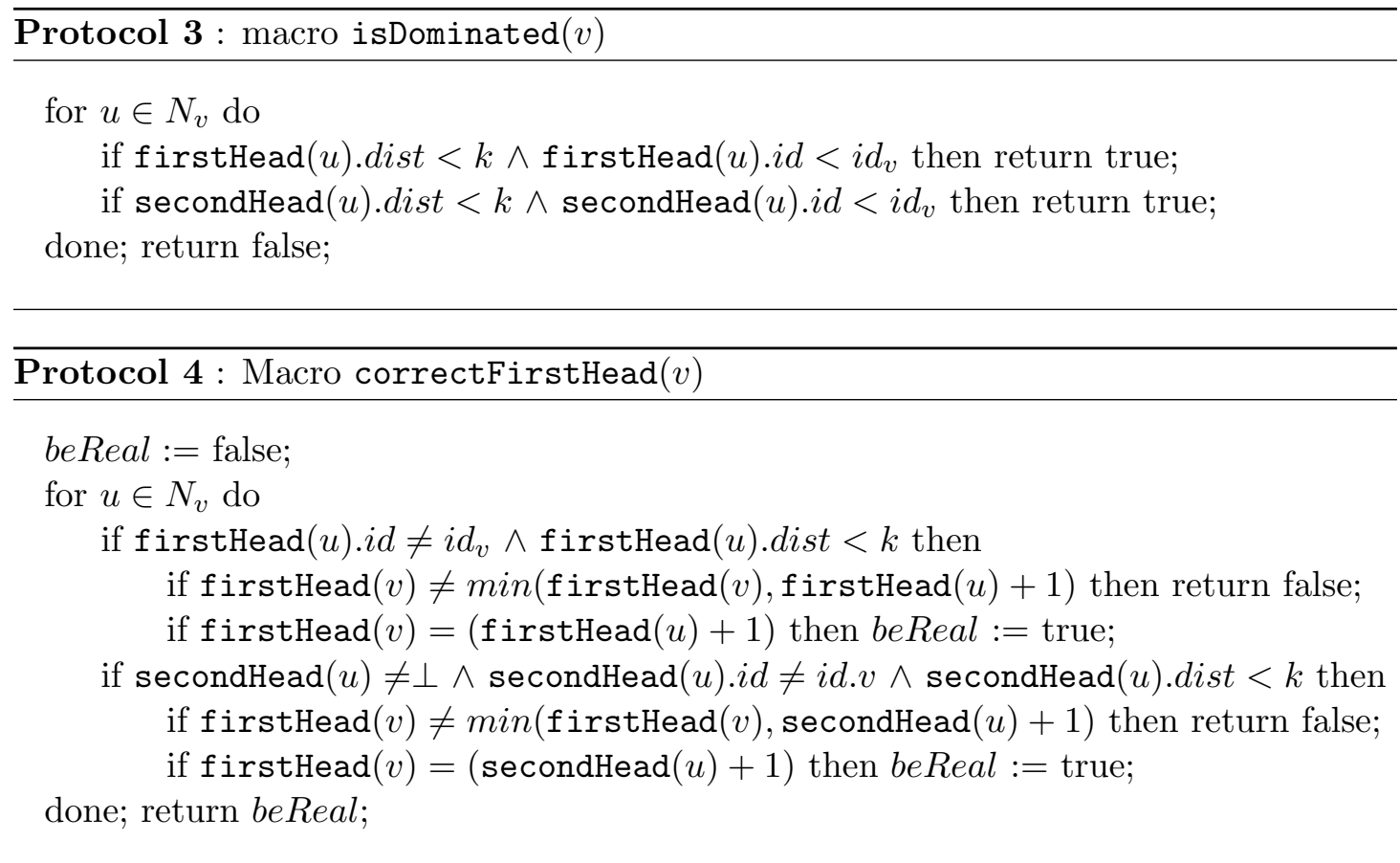

$\operatorname{secondHead}(v)$ is $\min (\mathrm{firstAugmentedIdSet}(v) \cup \perp)$; otherwise the macro returns false.

The macro computingSecondHead $(v)$ (defined in protocol 7) returns $\min ($ secondAugmentedIdSet $(v)$ ) if the set secondAugmentedIdSet $(v)$ is not empty; otherwise the macro returns $\perp$.

Once the system is stabilized, the set firstAugmentedIdSet $(v)$ ) contains some heads in $\mathrm{k}$-neighborhood of $v$. More precisely, this set contains the closest and second closest head to $v$ if there are in the $\mathrm{k}$-neighborhood of $v$. If the $k$ 's neighborhood of a node $v$ does not contain any head then the set firstAugmentedIdSet $(v))$ is empty. So the predicate isDefended $(v)$ is not verified. If $v$ is an ordinary node then $v$ is enabled (the rule $\mathbf{R E}$ or the rule $\mathbf{R U}$ is enabled). Therefore, the heads set is a $k$-dominating set, in a terminal configuration.

If toResign $(v)$ is verified then $v$ has in its k-neighborhood a head $u$ having a smaller identifier than $v$ 's identifier (i.e. $i d_{v}>i d_{u}$ ). In this case, if $v$ is a head, it is enabled. So, the set of heads is a $k$-independent set, in any terminal configuration.

The proof of the protocol $\mathcal{S I D}$ has two parts.

- In the section 4, we prove that a terminal configuration of $\mathcal{S I D}$ protocol is legitimate : the set of heads is a $k$-independent dominating set.

- In the section 5, we prove that all maximal computations under any unfair dis- 

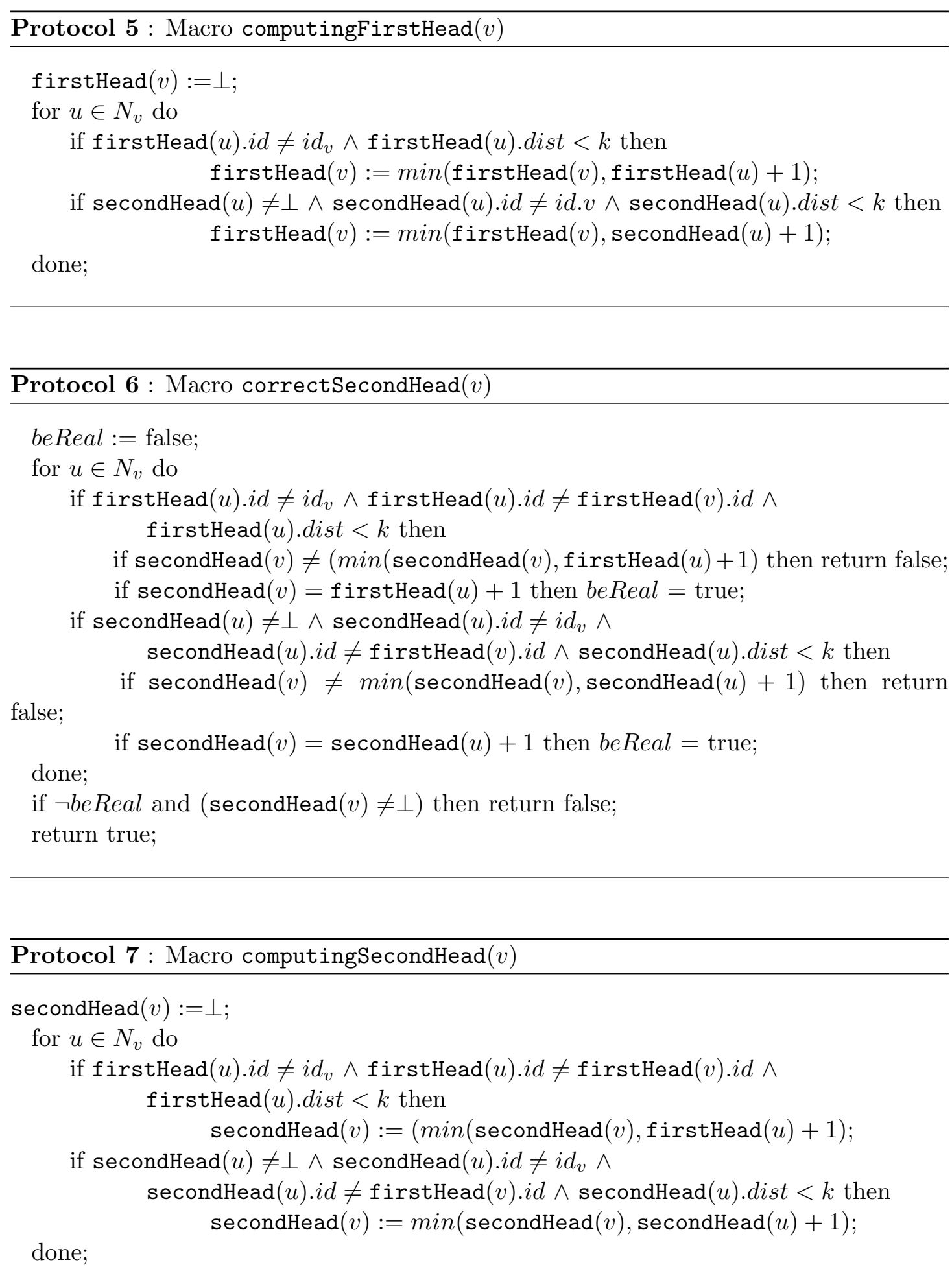
tributed scheduler are finite by reductio ad absurdam arguments.

\section{Correctness of the protocol $\mathcal{S I D}$}

In this section, we prove that all terminal configuration of $\mathcal{S I D}$ protocol are legitimate: the set of heads is a $k$-independent dominating set.

Definition 8 The property $\operatorname{Ordinary} \operatorname{Pr}(i)$ defined for all $i \in[1, k]$ is verifierd if the two following statements are satisfied:

- OrdinaryPrFirst (i): for all ordinary node $v$, firstHead $(v)=\left(i, i d_{u}\right)$ if and only if $u$ is the closest head to $v$ and $i$ is the distance between $u$ and $v$.

- OrdinaryPrSecond $(i)$ : for all node $v$, $\operatorname{secondHead}(v)=\left(i, i d_{w}\right)$ if and only if $w$ is the second closest head to $v$ and $i$ is the distance between $w$ and $v$.

Observation 1 In a terminal configuration,

1. An ordinary node $v$ does not verify OrdinaryToUpdate $(v)$;

so firstHead $(v)=\min ($ firstAugmentedIdSet $(v))$ and

$\operatorname{secondHead}(v)=\min (\operatorname{secondtAugmentedIdSet}(v) \cup \perp)$.

2. A head $u$ does not verify $\operatorname{HeadToUpdate}(u)$;

3. Let $w$ be a node (head or ordinary), firstHead $(w) \neq \perp$;

4. if $v$ is an ordinary node then firstHead $(v)$.dist $>0$;

5. if $\operatorname{secondHead}(v) \neq \perp$ then $\operatorname{secondHead}(v)$.dist $>0$;

6. if $\operatorname{secondHead}(v) \neq \perp$ then $\operatorname{secondHead}(v)$.dist $\geq$ firstHead $(v)$.dist because secondAugmentedIdSet $(v) \subset$ firstAugmentedIdSet $(v)$.

Lemma 2 In a terminal configuration of protocol $\mathcal{S I D}$, the property $\operatorname{OrdinaryPr}(1)$ is verified.

\section{Proof 2}

Let $v$ be an ordinary node, in a terminal configuration of protocol $\mathcal{S I D}$, named $c$.

Assume that $(1, x) \in$ firstAugmentedIdSet $(v)$. So $v$ has a neighbor $u$ such that firstHead $(u)=(0, x)$ or $\operatorname{secondHead}(u)=(0, x)$.

According to observation $1.4 \operatorname{secondHead}(u)$.dist $>0$ or $\operatorname{secondHead}(u)=\perp$. So $v$ has a neighbor $u$ such that $\mathrm{firstHead}(u)=(0, x)$. According to observation $1.3 \mathrm{u}$ is a head; so $x=i d_{u}$.

Notice that $\forall a \in$ firstAugmentedIdSet $(v)$, we have a.dist $>0$, in $c$.

Proof of OrdinaryPrFirst(1). If $v$ has a head at distance 1 then $v$ has a neighbor $u$ such that $\operatorname{firstHead}(u)=\left(0, i d_{u}\right)$. So, We have $\operatorname{firstHead}(v)=\left(1, i d_{u}\right)$ with $u$ being 
the head in v's neighborhood having the smallest identifier.

If $v$ has not a head at distance 1 then for any $u$ neighbor, we have firstHead(u).dist $>0$. and $\operatorname{secondHead}(u)$.dist $>0$ or $\operatorname{secondHead}(u)=\perp$ (according to observation 1.4). In this case, firstHead $(v)$.dist $>1$.

Proof of OrdinaryPrSecond(1). If $v$ has several heads at distance 1 then $v$ has a neighbor $w$ such that $\operatorname{firstHead}(w)=\left(0, i d_{w}\right)$ with $i d_{w} \neq \operatorname{firstHead}(v) . i d$. So, $\operatorname{secondHead}(v)=$ $\left(1, i d_{w}\right)$ with $w$ being the head in $v$ 's neighborhood having the second smallest identifier. If $v$ has at most one head at distance 1 then $v$ has not a neighbor $w$ such that firstHead $(w)=$ $\left(0, i d_{w}\right)$ with $i d_{w} \neq \mathrm{firstHead}(v) . i d$. In this case, $\operatorname{secondHead}(v)$.dist is larger than 1 or $\operatorname{secondHead}(v)=\perp$.

Lemma 3 Let $i$ be a positive integer smaller than $k$. In a terminal configuration of protocol $\mathcal{S I D}$, if the properties OrdinaryPr $(j)$ are verified for all $j \in[1, i]$ then the property Ordinary $\operatorname{Pr}(i+1)$ is verified.

Proof 3 Let us assume that the properties $\operatorname{Ordinary} \operatorname{Pr}(j)$ are verified for all $j \in[1, i]$ in any terminal configuration of protocol $\mathcal{S I D}$.

In a terminal configuration $c,(j, x) \in$ augmentedIdSet $(v)$ iff $v$ has a neighbor $u$ such that $\mathrm{firstHead}(u)=(j-1, x)$, or $\operatorname{secondHead}(u)=(j-1, x)$. If $j=1$ then $u$ is a head in $c$, according to Observation 1. If $1<j \leq i+1$ then $x$ is the identifier of a head in $c$ at distance $j-1$ of $u$, according to the property $\operatorname{Ordinary} \operatorname{Pr}(j-1)$. So $x$ is the identifier of a head at distance at most $j$ of $v$, in $c$.

Proof of OrdinaryPrFirst $(i+1)$. Let $v^{\prime}$ be the closest head to $v$ and $d^{\prime}$ the distance from $v^{\prime}$ to $v$ in the terminal configuration c. Assume that $0<d^{\prime} \leq i+1$. $v$ has a neighbor $u$ at distance $d^{\prime}-1$ to $v^{\prime}$. In $c$, the node $v^{\prime}$ is the closest head of $u$; so firstHead $(u)=\left(d^{\prime}-1, i d_{v^{\prime}}\right)$, according to the properties $\operatorname{Ordinary} \operatorname{Pr}\left(d^{\prime}-1\right)$. According to the properties $\operatorname{Ordinary} \operatorname{Pr}(j)$ forall $j \in[1, i]$, in $c$, we have the following properties,

- if $(l, i d) \in$ firstAugmentedIdSet $(v)$ then $l \geq d^{\prime}$; and

- if $\left(d^{\prime}, i d\right) \in$ firstAugmentedIdSet $(v)$ then $i d \geq i d_{v^{\prime}}$. In $c$,

We conclude that $\mathrm{firstHead}(v)=\left(d^{\prime}, i d_{v^{\prime}}\right)$, in $c$.

Proof of OrdinaryPrSecond $(i+1)$. Assume that the network has several heads. Let $v$ " be the second closest head to $v$ and $d$ " the distance from $v$ " to $v$, in a terminal configuration c. $v$ has a neighbor $u$ at distance $d "-1$ to $v "$ in $c$. (we have d" $>0$ ). v" is the first or second closest head to $u$, in $c$. Assume that d" $\leq i+1$. According to the property $\operatorname{Ordinary} \operatorname{Pr}\left(d^{\prime \prime}-1\right), \operatorname{firstHead}(u)=\left(d^{\prime \prime}-1, i d_{v}\right) \vee \operatorname{secondHead}(u)=\left(d "-1, i d_{v "}\right)$, in c. According to the properties $\operatorname{Ordinary} \operatorname{Pr}(j)$ forall $j \in[1, i)$, in $c$, we have the following properties,

- if $(l, i d) \in$ secondAugmentedIdSet $(v)$ then $l \geq d "$;

- if $\left(d^{\prime \prime}, i d\right) \in \operatorname{secondAugmentedIdSet}(v)$ then $i d \geq i d_{v "}$. 
We conclude that $\operatorname{secondHead}(v)=\left(d^{\prime \prime}, i d_{v^{\prime \prime}}\right)$.

The following corollary is a direct result of lemmas 2 and 3 . It establishes that the set of heads is a $k$-dominating set.

Corollary 1 Let $v$ be a ordinary node, in a terminal configuration of protocol SID. firstHead $(v)$.id is the closest head to $v$; their distance is $\operatorname{firstHead}(v)$.dist $\leq k$. If $\operatorname{secondHead}(v)=\perp$ then $v$ has a single head in its $k$-neigborhood; otherwise $\operatorname{secondHead}(v)$.id is the second closest head to $v$; their distance is $\operatorname{secondHead}(v)$.dist.

The following theorem establishes that the set of heads is a $k$-independent set.

Theorem 1 Let $v$ be a head, in a terminal configuration of protocol SID, named $c . v$ has not head in its k-neigborhood.

Proof 4 We will prove that if a head has another head in its $k$-neigborhood then the configuration $c$ is not terminal.

Let wrongHeadSet the set of heads having one or several heads in their k-neigborhood. Assume that wrongHeadSet is not empty. We denoted by $v 1$ the node of wrongHeadSet having the largest identifier. We denote by $v 2$, the closest head to $v 1$ and by $d$ the distance between $v 1$ and $v 2$. We have $0<d \leq k$ and $i d_{v 2}<i d_{v 1}$.

The node $v 1$ has a neighbor $u$ at distance $d-1$ of $v 2$. The node $v 2$ is the first or the second closest head to $u$. According to corollary $1,\left(d-1, i d_{v 2}\right)=$ firstHead $(u)$ or $\left(d-1, i d_{v 2}\right)=$ $\operatorname{secondHead}(u) . v 1$ is enabled because $v 1$ satisfied the predicate toResign $(v 1)$.

\section{Termination of the protocol $\mathcal{S I D}$}

In this section, we prove that all maximal computations of protocol $\mathcal{S I D}$ under any unfair distributed scheduler are finite by reductio ad absurdam arguments.

Let $e$ be a maximal computation starting from a configuration, named $c 0$. In a configuration $c$ reached by $e$, for all node $v, \operatorname{firstHead}(v)_{c} . i d$ is either the identifier of an node or this value appears in the initial configuration (i.e. there is a node $u$, such that firstHead $\left.(v)_{c} . i d=\operatorname{firstHead}(u)_{c 0} . i d \vee \operatorname{firstHead}(v)_{c} . i d=\operatorname{secondHead}(u)_{c 0} . i d\right)$. So, the value taken by a variable firstHead in $e$ belongs to a bounded set. Similary we prove also that the value taken by a variable secondHead in $e$ belongs to a bounded set.

\subsection{RR and RE rules}

Along any computation, a node performs at most one time the rule RC.

Assume that a or several nodes perform infinitely often the action $\mathbf{R E}$ or the action $\mathbf{R R}$. Between two consecutive actions $\mathbf{R E}$ by a node $u$, this node has performed on time the 
action $\mathbf{R R}$. So a node $u$ that infinitely often perform the action $\mathbf{R E}$ or the action $\mathbf{R R}$ changes its status infinitely often. We name $u^{+}$the node the smallest identifier among nodes that changes their status infinitely often. $e$ has a suffix $e 1$ where only nodes having a identifier larger than $i d_{u^{+}}$changes their status (i.e. to perform the action $\mathbf{R E}$ or the action $\mathbf{R R}$ ).

As the set of value taken by $\operatorname{firstHead}\left(u^{+}\right)$is bounded; along $e 1$, infinitely often after the action $\mathbf{R R}\left(u^{+}\right)$, firstHead $\left(u^{+}\right)$has the same value, denoted $(l+1, i d)$. Notice that $i d<i d_{u^{+}}$and $0<l<k$. So $u^{+}$has a neighbor $u_{l}$ such that, infinitely often before the action $\mathbf{R R}\left(u^{+}\right), u_{l}$ verfies firstHead $\left(u_{l}\right)=(l, i d)$ or $\operatorname{secondHead}\left(u_{l}\right)=(l, i d)$.

At time, where $u^{+}$becomes head, we have firstAugmentedIdSet $\left(u^{+}\right)=\emptyset$. So, the values of $u_{l}$ variables are infinitely often larger than $(l, i d)$ : So $u_{l}$ gives infinitely often to one of its variables the value $(l, i d)$, but also gives a larger value to the same variable.

Assume that $l>0$. At time where $u_{l}$ gives the value $(l, i d)$ to one of its variable : $u_{l}$ has a neigbor $u_{l-1}$, having the value $(l-1, i d)$. At time where $u_{l}$ gives a larger value than $(l, i d)$ to the same variable : $u_{l-1}$ has a larger value than $(l-1, i d)$. We conclude that there is a series of $l+1$ nodes : $u_{l}, u_{l-1}, . . u_{0}$ such that $u_{i}$ has infinitely often has the value $(i, i d)$ and infinitely often does not have this value along $e 1$.

Along $e 1, u_{0}$ performs infinitely often the action $\mathbf{R R}$ and the action $\mathbf{R E}$. We have $i d=i d_{u_{0}}<i d_{u^{+}}$: there is a contradiction.

So $e$ has a suffix, named $e 2$, in which the only rule performed is $\mathbf{R U}$.

\subsection{RU rule}

Assume that a node or several nodes changing infinitely often their value firstHead or their value secondHead along $e 2$. We named $\mathrm{min}^{+}$the smallest value infinitely often allocates to the variable firstHead or to the variable secondHead of one of these nodes. Let $e 3$ be the suffix of $e 2$ in which no variable firstHead and no variable secondHead gets a value smaller than $\min ^{+}$. Along $e 3$, infinitely often, a node, named $u^{+}$, performs RU action to set the value $\mathrm{min}^{+}$to its variable firstHead or its variable secondHead; and infinitely often, $u^{+}$performs $\mathbf{R U}$ action to set to the same variable a value larger than $\min ^{+}$.

Let $c \rightarrow c^{\prime}$ be a computation step of $e 3$ where $u^{+}$performs $\mathbf{R U}$ action to set a value larger than $\mathrm{min}^{+}$to its variable firstHead or to its variable secondHead. In $c, \mathrm{~min}^{+}$ is smaller than $\min \left(\mathrm{firstAugmentedIdSet}\left(u^{+}\right)\right.$) or $\mathrm{min}^{+}$is smaller than

$\min \left(\right.$ secondAugmentedIdSet $\left(u^{+}\right)$) . This property stays verified along $e 3: u^{+}$never sets the value $\mathrm{min}^{+}$to its variable firstHead (resp. to its variable secondHead). There is a contradiction.

We have established that $e 2$ has a suffix $e 4$ where no rule is executed. A terminal configuration is reached. 


\section{Conclusion}

A simple and silent self-stabilizing protocols building $k$-independent dominating sets is presented. The obtained $k$-independent dominating set contains at most $\lfloor(2 n) /(k+$ $2)\rfloor$ nodes. The protocol converges under the unfair distributed scheduler (the weakest scheduling assumption). The protocol is memory efficient : it requires only $2 \log ((k+$ 1) $n+1)+1$ bits per node.

The computation of the convergence time of the protocol $\mathcal{S I D}$ is opened question.

\section{References}

[1] D. Bein, A. K. Datta, C. R. Jagganagari, and V. Villain. A self-stabilizing linkcluster algorithm in mobile ad hoc networks. In International Symposium on Parallel Architectures, Algorithms and Networks (ISPAN'05), pages 436-441, 2005.

[2] A. Bui, S. Clavière, A. K. Datta, L. L. Larmore, and D. Sohier. Self-stabilizing hierarchical construction of bounded size clusters. In 18th International Colloquium Structural Information and Communication Complexity (SIROCCO'11), Springer LNCS 6796, pages 54-65, 2011.

[3] E. Caron, A. K. Datta, B. Depardon, and L. L. Larmore. self-stabilizing k-clustering algorithm for weighted graphs. Journal of Parallel and Distributed Computing, 70:1159-1173, 2010.

[4] A. Datta, S. Devismes, and L. Larmore. A self-stabilizing $o(n)$-round $k$-clustering algorithm. In 28th IEEE Symposium on Reliable Distributed Systems (SRDS'09), pages $147-155,2009$.

[5] A. K. Datta, L. L. Larmore, S. Devismes, K. Heurtefeux, and Y. Rivierre. Competitive self-stabilizing k-clustering. In IEEE 32th International Conference on Distributed Computing (ICDCS'12), pages 476-485, 2012.

[6] A. K. Datta, L. L. Larmore, S. Devismes, K. Heurtefeux, and Y. Rivierre. Selfstabilizing small k-dominating sets. International Journal of Networking and Computing, 3(1):116-136, 2013.

[7] A. K. Datta, L. L. Larmore, and P. Vemula. A self-stabilizing o(k)-time k-clustering algorithm. The Computer Journal, 53(3):342-350, 2010.

[8] M. Demirbas, A. Arora, V. Mittal, and V. Kulathumani. A fault-local self-stabilizing clustering service for wireless ad hoc networks. IEEE Trans. Parallel Distrib. Syst., 17(9):912-922, 2006.

[9] S. Dolev and N. Tzachar. Empire of colonies self-stabilizing and self-organizing distributed algorithms. In the 10th International Conference On Principles Of Distributed Systems (OPODIS'06), Springer LNCS 4305, pages 230-243, 2006. 
[10] V. Drabkin, R. Friedman, and M. Gradinariu. Self-stabilizing wireless connected overlays. In Conference On Principles Of Distributed Systems (OPODIS'06), Springer LNCS 4305, pages 425-439, 2006.

[11] W. Goddard, S. T. Hedetniemi, D. P. Jacobs, and P. K. Srimani. Self-stabilizing protocols for maximal matching and maximal independent sets for ad hoc networks. In the 5th IPDPS Workshop on Advances in Parallel and Distributed Computational Models (WAPDCM'03), 2003.

[12] C. Johnen. Fast self-stabilizing $k$-independant dominating set construction. Technical Report RR-1472-13, Univ. Bordeaux, LaBRI, UMR 3800, F-33400 Talence, France, June 2013.

[13] C. Johnen and F. Mekhaldi. Robust self-stabilizing construction of bounded size weight-based clusters. In the 16th International Euro-Par Conference (EuroPar'10), Springer LNCS 6271, pages 535-546, 2010.

[14] C. Johnen and L. H. Nguyen. Self-stabilizing construction of bounded size clusters. In the IEEE 8th International Symposium on Parallel and Distributed Processing and Applications (ISPA'08), pages 43-50, 2008.

[15] C. Johnen and L. H. Nguyen. Robust self-stabilizing weight-based clustering algorithm. Theoretical Computer Science, 410(6-7):581-594, 2009.

[16] S. Kamei and H. Kakugawa. A self-stabilizing approximation for the minimum connected dominating set with safe convergence. In the 12th Conference On Principles Of Distributed Systems (OPODIS'08), Springer, LNCS 5401, pages 496-511, 2008.

[17] A. Larsson and P. Tsigas. A self-stabilizing (k,r)-clustering algorithm with multiple paths for wireless ad-hoc networks. In IEEE 31th International Conference on Distributed Computing Systems, (ICDCS'11), pages 353-362. IEEE Computer Society, 2011.

[18] A. Larsson and P. Tsigas. Self-stabilizing (k,r)-clustering in clock rate-limited systems. In 19th International Colloquium Structural Information and Communication Complexity, (SIROCCO'12), Springer, LNCS 7355, pages 219-230, 2012.

[19] N. Mitton, E. Fleury, I. Guérin-Lassous, and S. Tixeuil. Self-stabilization in selforganized multihop wireless networks. In International Conference on Distributed Computing Systems Workshops (WWAN'05), pages 909-915, 2005.

[20] Z. Xu, S. T. Hedetniemi, W. Goddard, and P. K. Srimani. A synchronous selfstabilizing minimal domination protocol in an arbitrary network graph. In Proceedings of the 5th International Workshop on Distributed Computing (IWDC'03), Springer LNCS 2918, pages 26-32, 2003. 